\title{
Spin-orbit Coupled Bose-Einstein Condensate under Rotation
}

\author{
Xiao-Qiang $\mathrm{Xu}$ and Jung Hoon Han* \\ Department of Physics and BK21 Physics Research Division, \\ Sungkyunkwan University, Suwon 440-746, Korea
}

(Dated: September 10, 2018)

\begin{abstract}
We examine the combined effects of Rashba spin-orbit ( $\mathrm{SO}$ ) coupling and rotation on trapped spinor Bose-Einstein condensates (BECs). Nature of single particle states is thoroughly examined in the Landau level basis and is shown to support the formation of half-quantum vortex. In the presence of weak $s$-wave interactions, the ground state at strong SO coupling develops ring-like structures with domains whose number shows step behavior with increasing rotation. For fast rotation case, the vortex pattern favors triangular lattice, accompanied by the density depletion in the central region and weakened Skyrmionic character as the SO coupling is enhanced. Giant vortex formation is facilitated when SO coupling and rotation are both strong.

PACS numbers: 05.30.Jp, 03.75.Mn, 67.85.Fg, 67.85.Jk
\end{abstract}

Introduction.- Bose-Einstein condensates (BECs) are a fascinating testing ground for many interesting condensed matter physics phenomena such as the formation of vortices by rotation. For a single component condensate, if the rotation is fast enough, the vortices form a triangular (Abrikosov) lattice, indicating the entry of BECs into a quantum Hall regime where the lowest Landau level (LLL) physics dominates [1]. In the case of spinor condensates, the competition between inter- and intracomponent interactions was found to yield even richer vortex patterns such as square lattice, double-core lattice, and interwoven "serpentine" vortex sheet [2], or even giant Skyrmions [3] if the symmetry between the two components is broken.

A recent focus in cold atom physics is on the influence of "engineered" Rashba-type or Dresselhaus-type spinorbit (SO) coupling among spinor BECs induced by the so-called "synthetic non-Abelian gauge fields" [4]. The $\mathrm{SO}$ effects in cold atoms have received increasing attention theoretically [5-7] and its first experimental realization was recently reported [8]. Non-trivial new structures, such as stripe phase and half-quantum vortex, have been found or predicted, enriching the phase diagram of spinor BEC system [6, 9].

All existing studies of SO effect in spinor condensates refer to non-rotating case, and the aim of this paper is to study the combined effects of Rashba SO coupling and rotation on spinor BECs, focusing on spin- $1 / 2$ case as the prototype. Even the single particle aspect of the Rashba BEC Hamiltonian in the presence of rotation is not well understood theoretically, and we address these issues first. We use the Landau level (LL) expansion to examine the ground state of the single particle system by exact diagonalization, then move on to discuss the influences of weak $s$-wave collisional interactions. New features which are absent from pure Rashba, or pure rotational BECs, are observed.

Single Particle States.- We start with the single par- ticle Hamiltonian describing the trapped Rashba SO coupled system under rotation $H_{0}=\int \Psi^{\dagger}\left(\mathbf{p}^{2}+2 \kappa \mathbf{p}\right.$. $\boldsymbol{\sigma}) \Psi /(2 m)-\Omega_{z} L_{z}+\int \Psi^{\dagger} V(\mathbf{r}) \Psi$, where $\mathbf{p}=-i \hbar \boldsymbol{\nabla}$ with $m$ being the particle mass, $\boldsymbol{\sigma}$ are the $2 \times 2$ Pauli matrices, and $\kappa$ denotes the strength of SO coupling. Note that we make implicit the $2 \times 2$ unit matrix for p. $\Psi=\left(\psi_{\uparrow}, \psi_{\downarrow}\right)^{T}$ describes the two-component wave function. $\Omega_{z}$ stands for the rotational frequency and $L_{z}=\int \Psi^{\dagger}(\mathbf{r} \times \mathbf{p})_{z} \Psi$ is the canonical angular momentum, while the non-canonical part $\propto \kappa \mathbf{r} \times \boldsymbol{\sigma} / m$ can be compensated for an external spatially dependent Rabi coupling between the two components [10]. The trapping potential $V(\mathbf{r})=m \omega^{2}\left(x^{2}+y^{2}\right) / 2$ is introduced to keep the boundary condition realistic. We assume that the trapping frequency in the $z$ direction is much higher than those of transverse directions, so we may view our condensate as an effective two-dimensional system with the degree of freedom in the $z$ direction frozen out. With the proliferation of parameters in the problem, it is convenient to turn all quantities dimensionless by introducing a length scale $\sqrt{\hbar / m \omega}$ and an energy scale $\hbar \omega$. The dimensionless Hamiltonian thus becomes

$$
\begin{aligned}
H_{0}= & \frac{1}{2} \int|(-i \boldsymbol{\nabla}+\gamma \boldsymbol{\sigma}-b \hat{z} \times \mathbf{r}) \Psi|^{2} \\
& +\gamma b \int \Psi^{\dagger}(\hat{z} \times \mathbf{r} \cdot \boldsymbol{\sigma}) \Psi+\frac{1}{2}\left(1-b^{2}\right) \int r^{2} \Psi^{\dagger} \Psi
\end{aligned}
$$

Two dimensionless parameters $\gamma=\kappa / \sqrt{\hbar m \omega}$ and $b=$ $\Omega_{z} / \omega$ are introduced. Note that the second term of Eq. (1) is induced by the interplay between rotation and SO effects. We require $b<1$ in order to prevent the complete cancelation of the trapping potential by the centrifugal force.

The solution of the single particle Hamiltonian (1) is conveniently obtained from its second-quantized form which reads

$$
\hat{H}_{0}=(1+b) \hat{a}^{\dagger} \hat{a}+(1-b) \hat{c}^{\dagger} \hat{c}+i \gamma\left(\begin{array}{cc}
0 & \hat{a}^{\dagger}-\hat{c} \\
\hat{c}^{\dagger}-\hat{a} & 0
\end{array}\right),
$$

where operators are introduced as $\hat{a}=\frac{1}{2} z+\partial_{\bar{z}}, \hat{a}^{\dagger}=$ 


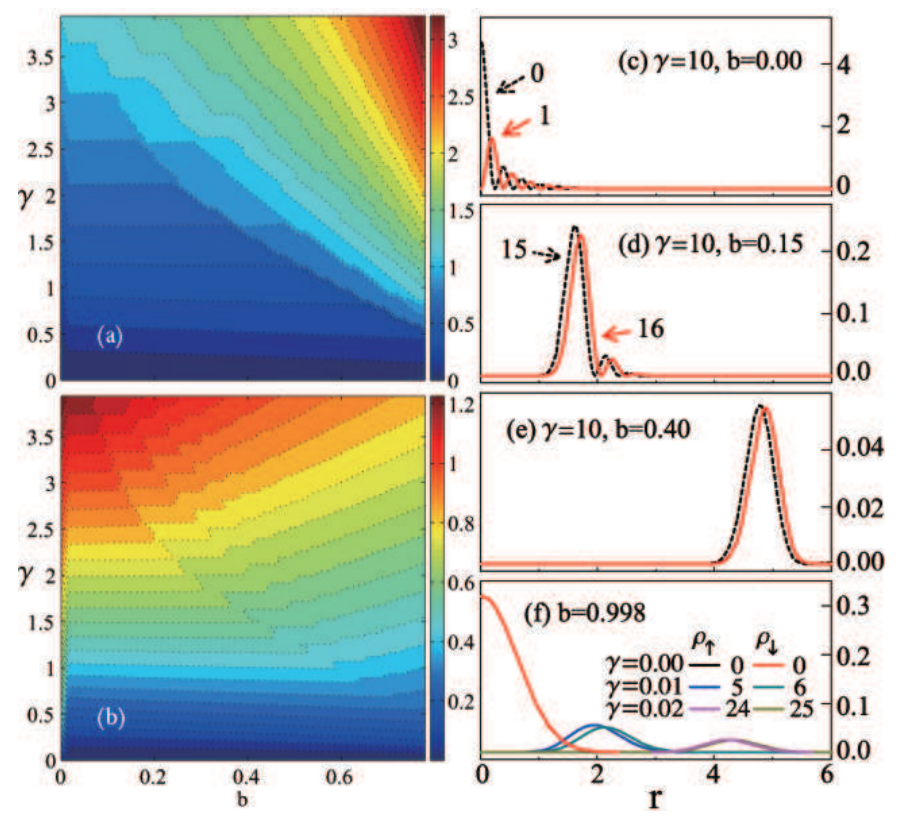

FIG. 1. (Color online) Single particle ground state of rotating Rashba-BEC Hamiltonian. (a) Average angular momentum $\left\langle\hat{c}^{\dagger} \hat{c}\right\rangle$ and (b) average LL index $\left\langle\hat{a}^{\dagger} \hat{a}\right\rangle$ for $\psi_{\uparrow}$ in $(b, \gamma)$ parameter space. Cubic roots $\left(\left\langle\hat{c}^{\dagger} \hat{c}\right\rangle^{1 / 3}\right.$ and $\left.\left\langle\hat{a}^{\dagger} \hat{a}\right\rangle^{1 / 3}\right)$ are plotted for improved view. (c) to (e) are radial density distributions of $\psi_{\uparrow}$ (black dotted) and $\psi_{\downarrow}$ (red solid) for the same large $\gamma$ and increasing $b$. The numbers with arrows denote the winding numbers of wave functions. (f) Densities of both components for the same $b$ and increasing $\gamma$. The digits in the legend label the winding numbers.

$\frac{1}{2} \bar{z}-\partial_{z}, \hat{c}=\frac{1}{2} \bar{z}+\partial_{z}$, and $\hat{c}^{\dagger}=\frac{1}{2} z-\partial_{\bar{z}}$ in complex coordinates $z=x+i y, \bar{z}=x-i y, \partial_{z}=\left(\partial_{x}-i \partial_{y}\right) / 2$, and $\partial_{\bar{z}}=\left(\partial_{x}+i \partial_{y}\right) / 2$. The two pairs of operators satisfy the bosonic commutation relations, $\left[\hat{a}, \hat{a}^{\dagger}\right]=\left[\hat{c}, \hat{c}^{\dagger}\right]=1$. We focus on positive $b$ and $\gamma$ without losing generality.

Without SO coupling, each Landau level state would be an eigenstate satisfying $\hat{a}^{\dagger} \hat{a} \phi_{n, m}=n \phi_{n, m}$ and $\hat{c}^{\dagger} \hat{c} \phi_{n, m}=m \phi_{n, m}$ with $n$ and $m$ being the Landau level index and angular momentum number. The eigenenergy is $\varepsilon_{0}=(1+b) n+(1-b) m$, i.e., $(1+b)$ and $(1-b)$ are the energy spacing between LLs and angular momentum states in each LL, respectively. The ground state is achieved for $n=m=0$. For nonzero $\gamma$, on the other hand, due to the specific form of Rashba term which couples neighboring LLs, one can use the ansatz that the eigenstate be formed as the linear combination $\Psi=\sum_{m \geq \nu}\left(x_{m} \phi_{m-\nu+1, m}, y_{m} \phi_{m-\nu, m}\right)^{T}$. By substituting $\Psi$ into $\hat{H}_{0} \Psi=\varepsilon \Psi$ with $\varepsilon$ being the eigenenergy, we find that the coefficients $x_{m}, y_{m}$ obey

$$
\begin{aligned}
& {[(1+b)(m-\nu+1)+(1-b) m] x_{m}} \\
& \quad+i \gamma\left(\sqrt{m-\nu+1} y_{m}-\sqrt{m+1} y_{m+1}\right)=\varepsilon x_{m} \\
& \quad[(1+b)(m-\nu)+(1-b) m] y_{m} \\
& \quad+i \gamma\left(\sqrt{m} x_{m-1}-\sqrt{m-\nu+1} x_{m}\right)=\varepsilon y_{m}
\end{aligned}
$$

The one-dimensional coupled linear equation set can be solved by numerical exact diagonalization with a reasonably large cutoff in $m$ for each $\nu$. Note that $\nu$ is an integer number defining the phase windings of $\psi_{\uparrow}$ and $\psi_{\downarrow}$ as $\nu-1$ and $\nu$, respectively, as shown in Fig. 1(c)-1(f). The difference by 1 indicates the formation of half-quantum vortex [11]. When either $b$ or $\gamma$ increases, the value of $\nu$ in the ground state increases correspondingly. In the limit $b=1$, each LL becomes infinitely degenerate, and we find by numerical approach the divergence of $\nu$ due to the disappearance of trapping potential.

In Fig. 1(a)-1(b) we show the average angular momentum $\left\langle\hat{c}^{\dagger} \hat{c}\right\rangle$ and average LL index $\left\langle\hat{a}^{\dagger} \hat{a}\right\rangle$ for $\psi_{\uparrow}\left(\psi_{\downarrow}\right.$ shows similar behavior). The visible steps in figures are strongly related to the discreteness of LLs. For fixed $\gamma$, increasing $b$ results in higher angular momentum and lower LL index due to the decrease of energy spacing between angular momentum states within each LL $(1-b)$ and the increase of energy spacing between LLs $(1+b)$. It also results in the more ring-like structure of the ground state density with increasing radius at larger $b$. By calculating the local spin density vector $\mathbf{n}=\Psi^{\dagger} \boldsymbol{\sigma} \Psi$, i.e., $n_{x}=$ $\psi_{\uparrow}^{*} \psi_{\downarrow}+\psi_{\downarrow}^{*} \psi_{\uparrow}, n_{y}=-i \psi_{\uparrow}^{*} \psi_{\downarrow}+i \psi_{\downarrow}^{*} \psi_{\uparrow}, n_{z}=\psi_{\uparrow}^{*} \psi_{\uparrow}-\psi_{\downarrow}^{*} \psi_{\downarrow}$, we also find that the spin texture at $b=0$ corresponds to the Skyrmion configuration which can be characterized by the Skyrmion density

$$
\rho_{s}(\mathbf{r})=\frac{1}{4 \pi} \frac{\mathbf{n} \cdot\left(\partial_{x} \mathbf{n} \times \partial_{y} \mathbf{n}\right)}{|\mathbf{n}|^{3}} .
$$

As $b$ increases, we observe the growing overlap between densities of two components, resulting in the decrease of $\rho_{s}$ due to $n_{z} \rightarrow 0$. Note that the Skyrmion density is strictly zero for any planar spin texture. Along with the density depletion in the trap center, the ground state exhibits spin spirals in the annular region.

When SO coupling is absent, $b \rightarrow 1^{-}$limit is associated with the quantum Hall regime. For small $\gamma$, we may view the SO coupling as a perturbation and the LLL picture still applies. However, when $\gamma$ increases to be comparable to $(1-b)$, higher angular momentum states contribute more and more to the ground state and the ring-like nature as well as a large winding number $\nu$ becomes dominant as seen in Fig. 1(f). For the value of $b$ we choose, when $\gamma$ is comparable to the LL energy spacing $(1+b)$, the radius becomes enormously large.

Interaction Effects.- We may now include interactions in the manner suitable for spin- $1 / 2$ condensate,

$$
H_{\mathrm{I}}=\frac{1}{4} \int\left[g_{c}\left(\rho_{\uparrow}+\rho_{\downarrow}\right)^{2}-g_{s}\left(\rho_{\uparrow}-\rho_{\downarrow}\right)^{2}\right],
$$

where $g_{c}=\left(g_{\uparrow \uparrow}+g_{\uparrow \downarrow}\right) N$ and $g_{s}=\left(g_{\uparrow \downarrow}-g_{\uparrow \uparrow}\right) N$ with $g_{\uparrow \uparrow}$ and $g_{\uparrow \downarrow}$ being the intra- and inter-component interactions, respectively. It is assumed that $g_{\uparrow \uparrow}=g_{\downarrow \downarrow}$ and $g_{\uparrow \downarrow}=g_{\downarrow \uparrow} . \quad N$ is the particle number, thus $\rho_{i}=\left|\psi_{i}\right|^{2}$ $(i=\uparrow, \downarrow)$ satisfy $\int \sum_{i} \rho_{i}=1$. The full Hamiltonian becomes $H=H_{0}+H_{\mathrm{I}}$. The ground state is numerically obtained using the imaginary time evolution method. Now 
we also consider two specific cases as in the single particle analysis above.
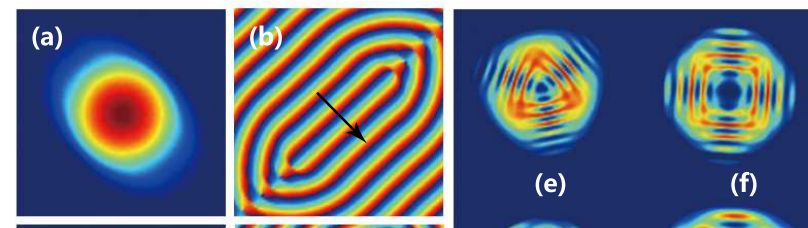

(e) (f)
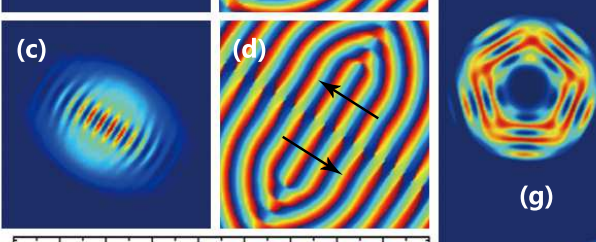

(g)

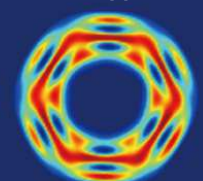

(h)
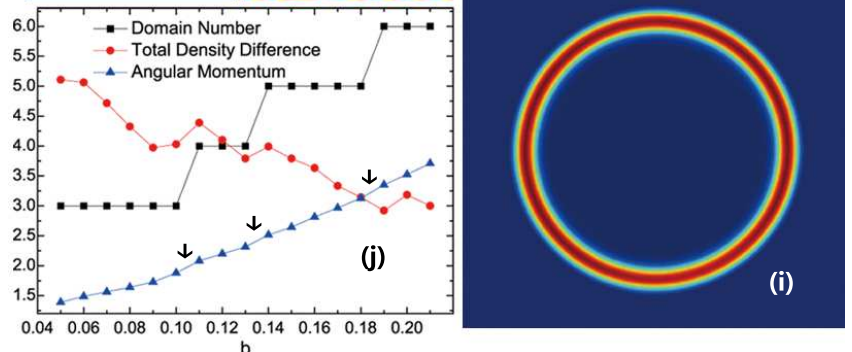

FIG. 2. (Color online) Ground state evolution with $b$ when $\gamma=10$ and $\left(g_{c}, g_{s}\right)=(9,-1)$. (a) and (b) are plots of the density $\left|\psi_{\uparrow}\right|^{2}$ and phase $\arg \left(\psi_{\uparrow}\right)$ for $b=0.0$, while (c) and (d) are for $b=0.02$. The long arrows in (b) and (d) represent the directions of $\mathbf{k}$ vectors. (e)-(i): Up-component densities $\left|\psi_{\uparrow}\right|^{2}$ for $b=0.08,0.12,0.16,0.20$, and 0.50 , respectively. (j) Dependence of domain number, total density difference $\int\left|n_{z}\right|$ ( $\times 10)$, and angular momentum of $\psi_{\uparrow}$ on $b . \psi_{\downarrow}$ shares similar evolution behavior.

We start with strong SO coupling case. When there is no rotation, the ground state is a stripe (ST) $\left(g_{s}>0\right)$ or a plane wave $(\mathrm{PW})\left(g_{s}<0\right)$ as was found recently [6]. In our trapped system, we choose weak interactions so as to recover the same stripe and $\mathrm{PW}$ phases. We first consider $g_{s}<0$ case and discuss $g_{s}>0$ later. The PW phase is composed of a single $\mathbf{k}$ state as shown in Fig. 2(a)-2(b). As $b$ increases gradually above zero, the $-\mathbf{k}$ state also begins to contribute, and each component of the condensate is divided into two parts with opposite momenta, indicated by long arrows in Fig. 2(d). Vortices emerge due to the counter-propagation of plane waves. Locations of these vortices also coincide with the low density regions shown in Fig. 2(c). We notice the similarity of this phase to the metastable state found in Ref. [6]. As we keep on increasing $b$, more $\mathbf{k}$ states contribute, and a single domain of vortex line (Fig. 2(c)) turns into several symmetrically placed domains with different k's (Fig. 2(e)-2(h)), while vortices nucleate at the center of the trap. The number of different $\mathbf{k}$ domains increases as $3 \rightarrow 4 \rightarrow 5 \rightarrow 6 \rightarrow \cdots$ until each component of the condensate becomes indistinguishable from a concentric ring (giant vortex) at strong enough rotation as shown in Fig. 2(i). The ring then expands in radius as $b$ further increases. The integer number of domains shows step behavior with rotation as shown in Fig. 2(j). Additionally, the angular momentum of the condensate increases monotonously with $b$, showing slight jumps (indicated by short arrows in Fig. $2(\mathrm{j})$ ) where the domain number changes. We also observe the enhanced overlap between densities of two components as $b$ increases. We define $\int\left|n_{z}\right|$ as the total density difference to quantify the overlap, where the absolute value is chosen to avoid cancelation among opposite signs of $n_{z}$ upon the integration. From Fig. 2(j) we observe the obvious overall trend of decrease, where the minor turns also coincide with the transition points of the domain number.
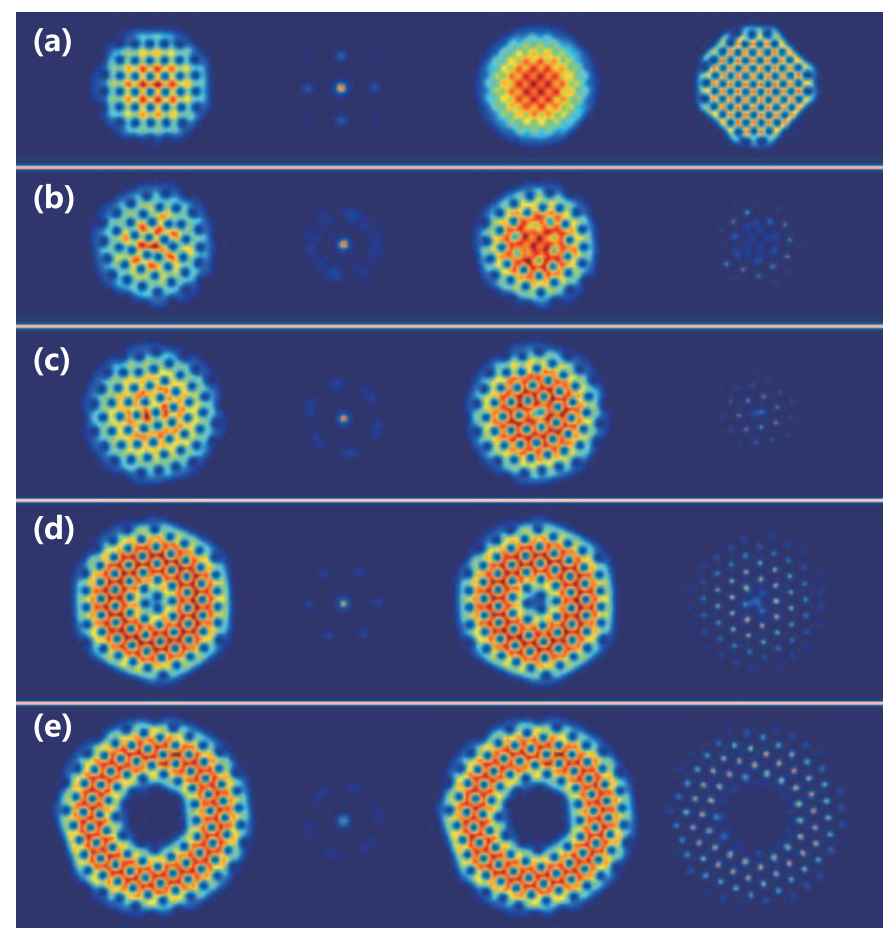

FIG. 3. (Color online) Ground state evolution with $\gamma$ for large rotation $b=0.998$ and $\left(g_{c}, g_{s}\right)=(9,-1)$. From left to right: density of up-component $\left|\psi_{\uparrow}\right|^{2}$, magnitude of its Fourier transformation $\left.\left|\int \exp [-i \mathbf{k} \cdot \mathbf{r}]\right| \psi_{\uparrow}\right|^{2} \mid$, total density of two components $\left(\left|\psi_{\uparrow}\right|^{2}+\left|\psi_{\downarrow}\right|^{2}\right)$, and the Skyrmion density $\rho_{s}$. (a)-(e): $\gamma=0.0,0.002,0.1,0.2$, and 0.3 , while the total Skyrmion number, defined as the spatial integral of Eq. (4), is $40.9143,19.2982,12.8454,8.1136$, and 3.7456 , respectively.

So far, we have discussed $g_{s}<0$ case. For $g_{s}>0$, the ground state is a stripe phase corresponding to the standing wave formed by two counter-propagating plane waves when $b=0$. As we increase rotation, we also observe similar evolution behavior as already shown in Fig. 2(c)-2(i). This implies that for weak interactions, as $b$ increases, the difference caused by the sign of $g_{s}$ is smeared out, giving way to the effects of large $\gamma$ and $b$.

When there is no SO coupling, the ground state in the so-called quantum Hall regime $\left(b \rightarrow 1^{-}\right)$is largely composed of the LLLs. Previous theories for the symmetrical 
case $\left(g_{\uparrow \uparrow}=g_{\downarrow \downarrow}\right)$ found an interlaced vortex array for each component, while the detailed lattice structures depend on the value of $g_{s}$ [2]. Starting from the known limit, $\gamma=0$, we hereby examine the influence of finite $\gamma$. For consistency, we choose the same values of interactions as in the strong SO coupling case and discuss the influence of $g_{s}$ later. Square vortex lattice $\left(g_{s} \rightarrow 0^{-}\right)$is shown in Fig. 3(a), along with the corresponding Skyrmion density $\rho_{s}(\mathbf{r})$. Interestingly, $\gamma=0$ lattice of displaced vortex cores of $\psi_{\uparrow}$ and $\psi_{\downarrow}$ already exhibits a regular pattern of Skyrmion density, qualifying it as a Skyrmion lattice [12]. We emphasize that the same Skyrmion charge is obtained for both spin-up and spin-down vortex cores.

From top to bottom of Fig. 3 we can find that, with increasing $\gamma$, the vortex lattice would be distorted first, and then densities deplete away from the trap center, forming the ring-like structure as in the single particle case. In the annular region of Fig. 3(d)-3(e) the vortices prefer to form triangular lattice pattern, even though we started with the square lattice for $\gamma=0$. Inside the trap the giant vortex is formed. During the transition from square to triangular lattice, we observe that the Skyrmion distribution shrinks first, and then expands as $b$ increases as shown in Fig. 3. To characterize the trend of the Skymrion density becoming more squeezed, we define the total Skyrmion number as $\int \rho_{s}$ which decreases correspondingly (see the caption of Fig. 3). This fact results from the increased overlap between up- and down-component densities. When the vortices overlap completely, Skyrmions are also destroyed.

Ground state of pure rotating spin- $1 / 2$ BECs may also be interlaced triangular vortex lattice when $g_{s}$ is close to $-g_{c}\left(g_{\uparrow \downarrow}\right.$ is small compared with $\left.g_{\uparrow \uparrow}\right)$, or double-core lattice when $g_{s}=0$, or even vortex sheet when $g_{s}>0$ [2]. For weak interactions, as we increase SO coupling, we observe similar evolution pattern as shown in Fig. 3 for all these vortex structures. Ring structure with a giant vortex about the trap center would appear, while triangular vortex lattices is favored in the annulus, and the overlap between densities would be enhanced. As in the case of strong SO coupling discussed above, the details of $g_{s}$ play no qualitative role for large $\gamma$ and $b$.

Summary.- We examined several prominent features of the rotating trapped Rashba BECs by analytical and numerical means. As we increase the rotational frequency, the system favors lower LLs with higher angular momenta, while the increase of SO coupling strength would drive the system to higher LLs with higher angular momentum. The two trends result from the competition between SO coupling strength and the energy spacings in the LL system. We also found the winding number difference by 1 between the two components indicating the formation of half-quantum vortex.

Weak interactions modify the single particle ground state dramatically. At strong SO coupling, the ring- structured densities develop multiple domains whose number shows step behavior as we increase the rotational frequency. At strong rotation, increasing SO coupling strength would favor triangular vortex lattice, deplete densities from the trap center, and weaken the Skyrmion crystal order. In the trap center the giant vortex is formed when SO coupling and rotation are both strong. Very recently in Ref. [13] we noticed the discussion of possible schemes for rotating SO coupled BEC system, exhibiting the challenge of experimental realization. Our findings are expected to build the understanding of the interplay between SO coupling and rotation.

H. J. H. is supported by Mid-career Researcher Program through NRF grant funded by the MEST (No. R012008-000-20586-0). We acknowledge helpful discussions and many insightful comments on our draft from Hui Zhai.

* Electronic address: hanjh@skku.edu

[1] D. A. Butts and D. S. Rokhsar, Nature (London) 397, 327 (1999); N. R. Cooper, S. Komineas, and N. Read, Phys. Rev. A 70, 033604 (2004); A. Aftalion, X. Blanc, and J. Dalibard, Phys. Rev. A 71, 023611 (2005).

[2] E. J. Mueller and T.-L. Ho, Phys. Rev. Lett. 88, 180403 (2002); T. Mizushima, K. Machida, and T. Kita, Phys. Rev. Lett. 89, 030401 (2002); T. Mizushima, N. Kobayashi, and K. Machida, Phys. Rev A 70, 043613 (2004); K. Kasamatsu, M. Tsubota, and M. Ueda, Phys. Rev. A 71, 043611 (2005).

[3] P. Mason and A. Aftalion, Phys. Rev. A 84, 033611 (2011).

[4] K. Osterloh, M. Baig, L. Santos, P. Zoller, and M. Lewenstein, Phys. Rev. Lett. 95, 010403 (2005); J. Ruseckas, G. Juzeliūnas, P. Öhberg, and M. Fleischhauer, Phys. Rev. Lett. 95, 010404 (2005); I. I. Satija, D. C. Dakin, and C. W. Clark, Phys. Rev. Lett. 97, 216401 (2006); L.-H. Lu and Y.-Q. Li, Phys. Rev. A 76, 023410 (2007); for a review, see J. Dalibard, F. Gerbier, G. Juzeliūnas, and P. Öhberg, arXiv:1008.5378.

[5] T. D. Stanescu, B. Anderson, and V. Galitski, Phys. Rev. A 78, 023616 (2008).

[6] C. Wang, C. Gao, C.-M. Jian, and H. Zhai, Phys. Rev. Lett. 105, 160403 (2010).

[7] Z. F. Xu, R. Lu, and L. You, Phys. Rev. A 83, 053602 (2011); T. Kawakami, T. Mizushima, and K. Machida, Phys. Rev. A 84, 011607 (2011) .

[8] Y.-J. Lin, K. Jiménez-Garcia, and I. B. Spielman, Nature 471, 83 (2011), and references therein.

[9] C. Wu, I. Mondragon-Shem, and X.-F. Zhou, Chin. Phys. Lett. 28, 097102 (2011); C.-M. Jian and H. Zhai, Phys. Rev. B 84, 060508(R) (2011).

[10] X.-F. Zhou, J. Zhou, and C. Wu, arXiv:1108.1238v1.

[11] G. E. Volovik, Exotic Properties of Superfluid ${ }^{3} \mathrm{He}$ (World Scientific, Singapore, 1992).

[12] V. Schweikhard, I. Coddington, P. Engels, S. Tung, and E. A. Cornell, Phys. Rev. Lett. 93, 210403 (2004).

[13] J. Radić, T. A. Sedrakyan, I. B. Spielman, and V. Galitski, arXiv:1108.4212v1. 